\title{
Analysis of oral microbiome from fossil human remains revealed the significant differences in virulence factors of modern and ancient Tannerella forsythia
}

Anna Philips', Ireneusz Stolarek', Luiza Handschuh', Katarzyna Nowis', Anna Juras'², Dawid Trzciński Wioletta Nowaczewska ${ }^{3}$, Anna Wrzesińska ${ }^{4}$, Jan Potempa ${ }^{5,6}$ and Marek Figlerowicz ${ }^{1,7^{*}}$

\begin{abstract}
Background: Recent advances in the next-generation sequencing (NGS) allowed the metagenomic analyses of DNA from many different environments and sources, including thousands of years old skeletal remains. It has been shown that most of the DNA extracted from ancient samples is microbial. There are several reports demonstrating that the considerable fraction of extracted DNA belonged to the bacteria accompanying the studied individuals before their death.

Results: In this study we scanned 344 microbiomes from 1000- and 2000-year-old human teeth. The datasets originated from our previous studies on human ancient DNA (aDNA) and on microbial DNA accompanying human remains. We previously noticed that in many samples infection-related species have been identified, among them Tannerella forsythia, one of the most prevalent oral human pathogens. Samples containing sufficient amount of $T$. forsythia aDNA for a complete genome assembly were selected for thorough analyses. We confirmed that the $T$. forsythia-containing samples have higher amounts of the periodontitis-associated species than the control samples. Despites, other pathogens-derived aDNA was found in the tested samples it was too fragmented and damaged to allow any reasonable reconstruction of these bacteria genomes. The anthropological examination of ancient skulls from which the $T$. forsythia-containing samples were obtained revealed the pathogenic alveolar bone loss in tooth areas characteristic for advanced periodontitis. Finally, we analyzed the genetic material of ancient $T$. forsythia strains. As a result, we assembled four ancient T. forsythia genomes - one 2000- and three 1000-year-old. Their comparison with contemporary $T$. forsythia genomes revealed a lower genetic diversity within the four ancient strains than within contemporary strains. We also investigated the genes of $T$. forsythia virulence factors and found that several of them (KLIKK protease and bspA genes) differ significantly between ancient and modern bacteria. (Continued on next page)
\end{abstract}

\footnotetext{
* Correspondence: marekf@ibch.poznan.pl

'Institute of Bioorganic Chemistry, Polish Academy of Sciences, 61-704 Poznan, Poland

${ }^{7}$ Institute of Computing Science, Poznan University of Technology, 60-965 Poznan, Poland

Full list of author information is available at the end of the article
}

C C The Author(s). 2020 Open Access This article is licensed under a Creative Commons Attribution 4.0 International License, which permits use, sharing, adaptation, distribution and reproduction in any medium or format, as long as you give appropriate credit to the original author(s) and the source, provide a link to the Creative Commons licence, and indicate if changes were made. The images or other third party material in this article are included in the article's Creative Commons licence, unless indicated otherwise in a credit line to the material. If material is not included in the article's Creative Commons licence and your intended use is not permitted by statutory regulation or exceeds the permitted use, you will need to obtain permission directly from the copyright holder. To view a copy of this licence, visit http://creativecommons.org/licenses/by/4.0/ The Creative Commons Public Domain Dedication waiver (http://creativecommons.org/publicdomain/zero/1.0/) applies to the data made available in this article, unless otherwise stated in a credit line to the data. 
(Continued from previous page)

Conclusions: In summary, we showed that NGS screening of the ancient human microbiome is a valid approach for the identification of disease-associated microbes. Following this protocol, we provided a new set of information on the emergence, evolution and virulence factors of T. forsythia, the member of the oral dysbiotic microbiome.

Keywords: aDNA, Ancient genomics, T. forsythia, Oral microbiome, Comparative genomics

\section{Background}

Currently, periodontitis is a common condition that affects approximately $15-20 \%$ of the worldwide population (age 35-44 years; WHO. 2012. Fact sheet N318: oral health. WHO, Geneva, Switzerland). This prevalence correlates well with the prevalence of periodontitis in adults (aged > 30 years) in the United States, at $46 \%$, with $8.9 \%$ having severe disease [1]. The molecular pathogenicity of periodontitis as a microbiota-shift disease is still far from fully understood [2]. According to a well-accepted paradigm, the disease is driven by dysbiotic bacterial flora composed of the red complex oral bacteria (Porphyromonas gingivalis, Treponema denticola and $T$. forsythia) as well as a cohort of newly recognized periodontal pathogens [3]. In a subgingival biofilm, they form a tightly knit community engaged in competitive and cooperative interactions [4]. A futile attempt of the host to eradicate dysbiotic biofilm fuels a chronic inflammatory reaction in the infected periodontium. In genetically susceptible hosts, this inflammation leads to dissolution of the periodontal ligament, alveolar bone resorption, deep periodontal pocket formation and eventual tooth loss [5].

Among recognized pathogens, T. forsythia is grossly under investigated, and only a handful of its virulence factors have been characterized to date [6]. This lack of knowledge is perplexing in light of a growing body of evidence that $T$. forsythia is strongly associated with periodontitis and must largely contribute to the pathogenicity of the microbiota in subgingival plaque $[4,7,8]$. To date, several virulence factors of $T$. forsythia have been reported [6]. The list of them is still growing and includes: (i) proteases (KLIKK, PrtH) $[9,10]$ that protect the bacterium from being killed by complement and bactericidal peptides [11-13]; (ii) dipeptidyl peptidase IV (DppIV) that is implicated in host tissue destruction [14, 15]; (iii) miropin that acts as a bacterial inhibitor of host broad-range proteases, some of them contributing to antibacterial activity of the inflammatory milieu [16]; (iv) glycosidases (SusB, SiaHI, NanH, and HexA) that degrade oligosaccharides and proteoglycans in saliva, gingival and periodontal tissues and promote disease progression [17-20]; and (v) the OxyR protein responsible for biofilm activity that facilitates and/or prolongs bacterial survival in diverse environmental niches [21].
Alike $P$. gingivalis, $T$. forsythia uses a type IX secretion system (T9SS) composed of PorK, PorT, PorU, Sov and several other conserved proteins to deliver virulence factors to the bacterial surface [22]. The T9SS cargo includes KLIKK proteases, BspA protein and components of the semi-crystalline S-layer (TfsA and TfsB). The latter provides bacteria with a protective shielding and promotes microbe adhesion [23, 24]. In addition, these proteins are heavily glycosylated with a unique complex O-linked decasaccharide containing nonulosonic acids, either legionaminic acid (Leg) or pseudaminic acid (Pse), a sialic acid-like sugars implicated in evasion of the host immune response. Of note, the occurrence of Leg or Pse is strain-specific $\{$ Bloch, $2019 \# 649$ \}. Among the surfaceanchored proteins, BspA is currently the best characterized T. forsythia virulence factor. BspA was shown to be involved in binding to fibronectin and fibrinogen [25]; to mediate interactions with other bacteria (among others with $T$. denticola [26]) and to induce bone loss in mice [26]. BspA belongs to the family of leucine-rich repeat (LRR) proteins. It is composed of 20 tandem LRR domains in the $\mathrm{N}$-terminal region and 4 immunoglobulinlike (Ig-like) domains typically found in bacteria. The LRR region plays a role in protein-protein interactions. The function of BspA Ig-like domains has not yet been determined, but it is suggested that they may stabilize the tertiary structure of LRRs [6]. Sequencing of the $T$. forsythia ATCC 43037 genome, apart from bspA (BFO_ RS14480), revealed five more genes (BFO_RS14345 (bspB), BFO_RS08355, BFO_RS14330, BFO_RS14330, and BFO_RS14330) encoding putative BspA-like proteins. Among them, BspB requires special attention because, in contrast to other BspA-like proteins, it possesses both LRR and Ig-like domains. While the amino acid sequence of the LRR region of BspA and $\mathrm{BspB}$ is different to a large extent, the Ig-like regions displayed 99\% amino acid sequence similarity. The BspB protein was identified in the $T$. forsythia outer membrane proteome, but its function is still unknown [27].

In earlier studies, we identified a wide spectrum of bacterial species in 1000- and 2000- year-old human remains and showed that some of them most likely accompanied their hosts before their deaths [28]. Here, we analyze the compositions of the ancient oral microbiomes. We used T. forsythia presence as a marker for 
the potential occurrence of periodontitis and showed statistically significant differences in the amount of DNA of periodontitis-associated bacteria in samples with the highest amounts of $T$. forsythia ancient DNA (aDNA) comparing to the reference samples. We also attempted a complete genome assembly of $T$. forsythia as three samples contained sufficient amounts of aDNA derived from this bacterium. Subsequently, we investigated the evolution of the T. forsythia genome, particularly focusing on genes encoding bacterial virulence factors that contribute to periodontitis development. To date, little is known about the genetic diversity of this common pathogen, especially in the context of its infectionassociated genes. Comparative studies of whole $T$. forsythia ancient and modern genomes, which we performed for the first time, shed light on this matter, revealing huge sequence variability in some virulencerelated genes. Moreover, the Roman Iron Age and medieval genomes analyses brought important information on the origin and evolution of this bacterium through ages and, most importantly, on the evolutionary conservation of its virulence factors.

\section{Results}

While examining 1000-2000-year old human skulls we found that some of them had typical for periodontitis pathogenic alveolar bone lesions in tooth areas. This observation roused the question whether it is possible to determine the nature of these lesions by identifying DNA biomarkers of periodontal infection in the oral microbiome collected from the ancient human remains. To this end, we scanned next-generation sequencing (NGS) metagenomic datasets obtained for 344 human skeletal remains by mapping reads to the database of unique clade-specific marker sequences [29]. The metagenomic dataset was generated with DNA extracted from human teeth dated from the 1st to the sixteenth c. AD. The biological material came from archaeological sites distributed across Poland.

An analysis of NGS data generated separately for each of the 344 studied individuals allowed us to estimate that nine samples contained more than $3 \%$ of $T$. forsythia DNA (Supplementary Table 1, [29]). The NGS datasets obtained for these nine samples were mapped against the T. forsythia reference genome (NC_016610.1), showing that for three out of nine samples, the average nucleotide coverage was $>5$, and for more than $80 \%$ of the $T$. forsythia genome, the nucleotide coverage was $\geq 3$ (Supplementary Figure $1 \mathrm{~A}$ ). These three samples were selected for further analyses. Additionally, the studied group was extended with the published data on the teeth microbiome of an individual living in Dalheim, Germany in the 10th-twelfth c. AD in which sample T. forsythia was also identified [30].

\section{Identification of periodontitis-associated bacteria in ancient samples}

Anthropological analyses revealed that all four skulls from which $T$. forsythia DNA was isolated had characteristic for periodontitis lesions in the tooth area (see Supplementary Material "Anthropological description of analyzed individuals" and Supplementary Figure 2 for PCA0088, PCA0198, and PCA0332 and [30] for G12). Accordingly, we investigated the overall microbial content of these DNA samples to determine the presence of other bacterial species that have been shown to be associated with periodontitis in humans [31]. Metagenomic analysis involving MetaPhlAn2 [29] revealed that bacteria consisted of $82.05,99.57,99.44,98.49 \%$ of the PCA0088, PCA0198, PCA0332 and G12 samples, respectively. The microbial content of each sample is shown in Supplementary Figure 7. Overall, 1 archaeal and 21 bacterial classes were identified. In the PCA0088 and PCA0332 samples, Clostridia was the most abundant class, consisting of 35.50 and $25.43 \%$ of all bacteria, respectively. In the PCA0198 sample, Actinobacteria was the majority, consisting of $28.74 \%$ of all bacteria; in G12, Bacilli constituted $27.99 \%$ of the bacterial component. The Bacteroidetes class, to which T. forsythia belongs, comprised 3.06, 4.49, 3.97, and 3.02\% of all bacteria in PCA0088, PCA0198, PCA0332, and G12, respectively. The characteristics of the genera identified within all of the classes uncovered the prevalence of taxa typical of human flora (85.09, 96.23, 97.48, and $96.05 \%$ in PCA0088, PCA0198, PCA0332, and G12), including $75.34,77.63,90.2$, and $81.04 \%$ of oral genera, respectively. The remaining genera consisted of ubiquitous environmental taxa typical of a wide range of soils and waters. At the species level, T. forsythia accounted for $5.91,22.76,4.23$ and $2.14 \%$ of bacterial species in the samples PCA0088, PCA0198, PCA0332, and G12, respectively, and was the most abundant species in the sample PCA0198. P. gingivalis and T. denticola, which together with $T$. forsythia constitute the "red complex", were detected in all four ancient samples. $P$. gingivalis represented $0.45,3.62,1.07$, and $0.35 \%$ of bacteria, and T. denticola represented 1.52, 2.95, 3.31, and $0.78 \%$ of bacteria in PCA0088, PCA0198, PCA0332, and G12, respectively. Additionally, we discovered in at least one of the four samples the following periodontitis-associated species: Filifactor alocis, T. medium, T. vincentii, Lachnospiraceae oral taxon 107, P. intermedia or G. elegans.

To check whether the amount of pathogenic species in the four analyzed samples differed from that in other ancient samples, we compared the microbial content of PCA0088, PCA0198, PCA0332, and G12 with the content found in the other 17 ancient samples in which human oral species consisted of $>75 \%$ [28] (Supplementary Table 5). The comparison of the content of 
periodontitis-associated species showed statistically significant differences. In particular, T. medium and T. vincentii abundances revealed the highest statistical significance (t-test, $p$-val $<0.0001)$ followed by $P$. intermedia and $P$. gingivalis abundances (t-test, $p$-val $<0.01$ ) and by T. forsythia and G. elegans abundances (t-test, $p$ val $<0.05$ ). It must be pointed out that there was no anthropological information on the inflammatory lesions on the 17 skeleton jaws from which the aDNA samples served in this analysis as references. Therefore, it is likely that the reference set contained samples derived from periodontitis sites. If so, this will only strengthen the significant difference in composition and abundance of periodontopathogenic species in the analyzed sets of samples.

\section{Assembly of the ancient $T$. forsythia genomes}

Based on NGS data obtained for the samples selected for the study (samples with the high levels of $T$. forsythia aDNA, we were able to assemble four variants of the full-length $T$. forsythia genome. Each variant was named after the sample IDs from which aDNA was isolated: PCA0088, PCA0198, and PCA0332. Sample PCA0088 was obtained from an individual buried in Masłomęcz during the Roman Iron Age (2nd-fourth c. AD) [32, 33], sample PCA0198 was from an individual living in Ląd during the Early Medieval Age (10th-twelfth c. AD, Supplementary Figure 3) [34], sample PCA0332 was from an individual living in Ostrów Lednicki during the Medieval Age (12th-thirteenth c. AD) and sample G12 was from an individual living in Dalheim, Germany in the 10th-twelfth c. AD [30]. Overall, 321,886, 444,721,
427,421, and 325,568 unambiguous reads from the PCA0088, PCA0198, PCA0332, and G12 samples, respectively, were mapped to the reference genome sequence (NC_016610.1), with average coverage of 7.03, 9.81, 9.71, and 6.95, respectively (Fig. 1a, Supplementary Table 1). To verify whether the assembled T. forsythia genomes were of ancient origin and whether the bacteria accompanied the humans before death, we analyzed the signatures of age-related DNA damage. aDNA damage patterns were evaluated using mapDamage2.0, which simulates the posterior distribution of deamination in DNA [35]. The analysis of reads mapped to the T. forsythia reference genome revealed typical aDNA damage patterns, as presented in Fig. 1b. An increase of $\mathrm{C}>\mathrm{T}$ (and G > A) nucleotide transition frequencies up to $25 \%$ at the 5' (and 3') end of DNA fragments was observed. Sample G12 was not included in this analysis because the polymerase that was used for library preparation (Phusion Finnzymes [30];) is not able to replicate through uracil; thus, age-related DNA modifications could not be assessed [36]. The length distribution of mapped reads (Supplementary Figure 1 B) showed that the $T$. forsythia average aDNA fragment was 82 , 85 and $88 \mathrm{nt}$ long in PCA0088, PCA0198, and PCA0332, respectively. As a single-end approach was applied for G12 sequencing, which does not allow read merging, the average read length of this sample $(73 \mathrm{nt})$ could not be directly compared with the read lengths of pair-end libraries used in our study. In summary, the read length distribution and DNA damage patterns supported the ancient origin of the sequenced T. forsythia DNA.

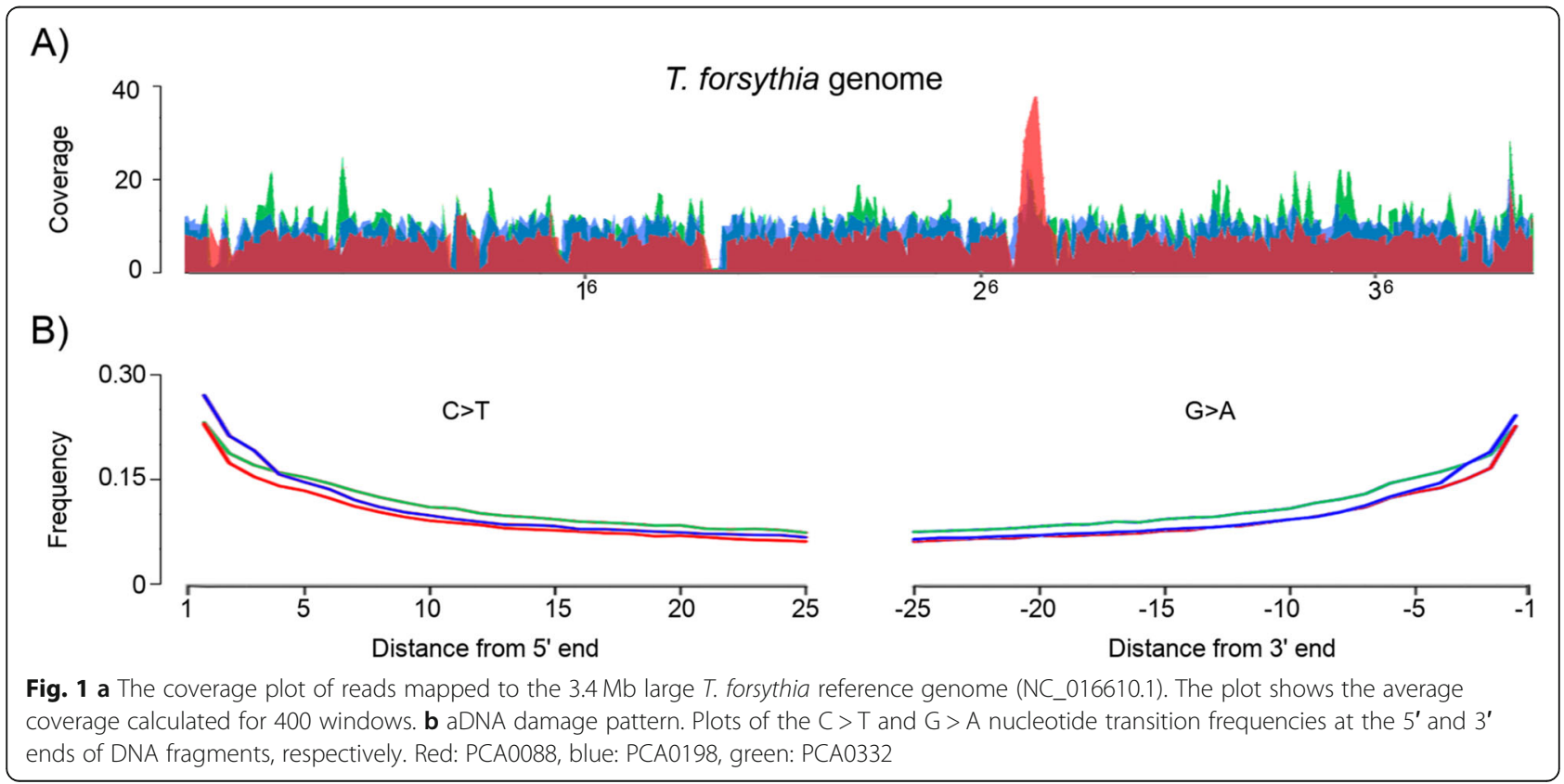


Comparative analysis of the reconstructed and modern $T$. forsythia genomes

To investigate contemporary and ancient $T$. forsythia genome diversity, we extended the studied group of four ancient genomes with ten publicly available modern genomes of $T$. forsythia (Table 1 ). First, we analyzed single nucleotide polymorphisms (SNPs, GATK tools [37]) and created an SNP-based phylogenetic tree with FastTree [38]. Second, we determined the deletion distribution.

The analysis involving the $T$. forsythia reference genome (92A2) showed that Roman Iron Age T. forsythia PCA0088 had 1167 SNPs, while in the medieval $T$. forsythia PCA0198, PCA0332 and G12 genomes, we identified 2645, 1933 and 1374 SNPs, respectively. For each modern $T$. forsythia genome, an average of $\sim 25$, 000 SNPs were identified. The relatively low number of SNPs identified in the ancient strains can be explained by incompleteness of the genomes, age-related aDNA modifications, restricted criteria of SNP calling as well as by their location on the SNP tree (see below). Generally, most SNPs were identified within known genes (92.58\%), including $1.33 \%$ of SNPs in virulence-associated genes. That yields, on average, 21 SNPs per gene and 28 SNPs per virulence factor gene (Supplementary Table $2 \mathrm{~A}$ ).

A SNP-based phylogenetic tree of $T$. forsythia contemporary genomes was constructed based on 64,413 SNPs that were discovered in at least one of the analyzed genomes, and the reference nucleotide was determined for all of the remaining modern genomes (Supplementary Table 2 A). Two Japanese genomes (KS16 and 3313) grouped together on the phylogenetic tree; however, the positions of genomes isolated in London (NSLK and NSLJ) and those obtained in the same location in the USA (UB4, UB20, and UB22) did not correlate with their geographical regions. Ancient T. forsythia genomes were subsequently "projected" (with pplacer [39]) on the previously generated tree of modern genomes (Fig. 2). We did not include ancient genomes during the construction of the initial phylogenetic tree, as the number of SNPs determined for the four ancient genomes was $\sim 10$-fold lower than the average number of SNPs identified in the contemporary genomes. This approach ensured that the similarity of ancient and contemporary $T$. forsythia was not caused by the reduced number of identified SNPs. All four ancient genomes were placed within the 92A2, 9610 and UB22 cluster. The oldest T. forsythia genome, PCA0088, which is geographically distinct from the three medieval genomes, displayed the closest relationship with 92A2. Further, to confirm the placement of ancient $T$. forsythia on the SNP phylogenetic tree, we repeated the analysis, but with more restricted criteria of nucleotide calling in the ancient genomes. To call a $\mathrm{SNP} /$ reference nucleotide, at least 10 -fold coverage was required (instead of the initially used 3-fold coverage, Supplementary Figure 4 A). Despite the use of more restrictive criteria, the general location of the four ancient genomes in the phylogenetic tree was the same. They remained within the 92A2, 9610 and UB22 cluster, though PCA0198 was placed closest to 92A2, whereas PCA0088 and G12 were the most distant. These differences, however, might be caused by the reduced number of SNPs, which was especially meaningful to the two ancient genomes with the lowest genome coverage (PCA0088 and G12). In the third attempt to construct the phylogenetic tree, we used a threshold of min. 3-fold coverage, and we also excluded SNPs identified in reads $<70$ nt long (Supplementary Figure 4 B) because Green et al. [40] showed that shorter reads containing a SNP are more likely to be unmapped because they carry less information to place them uniquely in the genome. In the next attempt, we excluded all $\mathrm{C} / \mathrm{T}$ and G/A SNPs identified in the PCA0088, PCA0198, PCA0332, and G12 genomes, as transitions $\mathrm{C}>\mathrm{T}$ and $\mathrm{G}>\mathrm{A}$ caused by DNA damage could be misidentified as original SNPs (Supplementary Figure $4 \mathrm{C}$ ). The ancient genomes again positioned closest to the 92A2 genome and arranged in the same way in both SNP trees (Supplementary Figure $4 \mathrm{~B}, \mathrm{C})$. In comparison to their locations in the original

Table 1 The list of contemporary T. forsythia strains with sequenced genomes

\begin{tabular}{|c|c|c|c|c|c|}
\hline Strain & NCBI BioProject id & Source & Location & Assembly & SNPs \\
\hline $92 \mathrm{~A} 2$ - the reference & PRJNA319 & Human periodontal pocket & USA, Massachusetts & Complete genome & N/A \\
\hline 3313 & PRJDB1007 & Human oral cavity & Japan, Tokyo & Complete genome & 26,310 \\
\hline KS16 & PRJDB1008 & Human oral cavity & Japan, Tokyo & Complete genome & 25,880 \\
\hline NSLJ & PRJNA401301 & Human Subgingival plaque & UK, London & Contig & 24,171 \\
\hline NSLK & PRJNA401301 & Human Subgingival plaque & UK, London & Contig & 25,744 \\
\hline ATCC 43037 & PRJNA548889 & Human periodontal pocket & USA, N/K & Scaffold & 26,806 \\
\hline UB20 & PRJEB15383 & Human Subgingival plaque & USA, New York & Scaffold & 24,845 \\
\hline UB22 & PRJEB15383 & Human Subgingival plaque & USA, New York & Scaffold & 21,796 \\
\hline UB4 & PRJEB15383 & Human Subgingival plaque & USA, New York & Scaffold & 27,183 \\
\hline 9610 & PRJNA340021 & Human periodontal pocket & USA, Washington & Scaffold & 24,232 \\
\hline
\end{tabular}




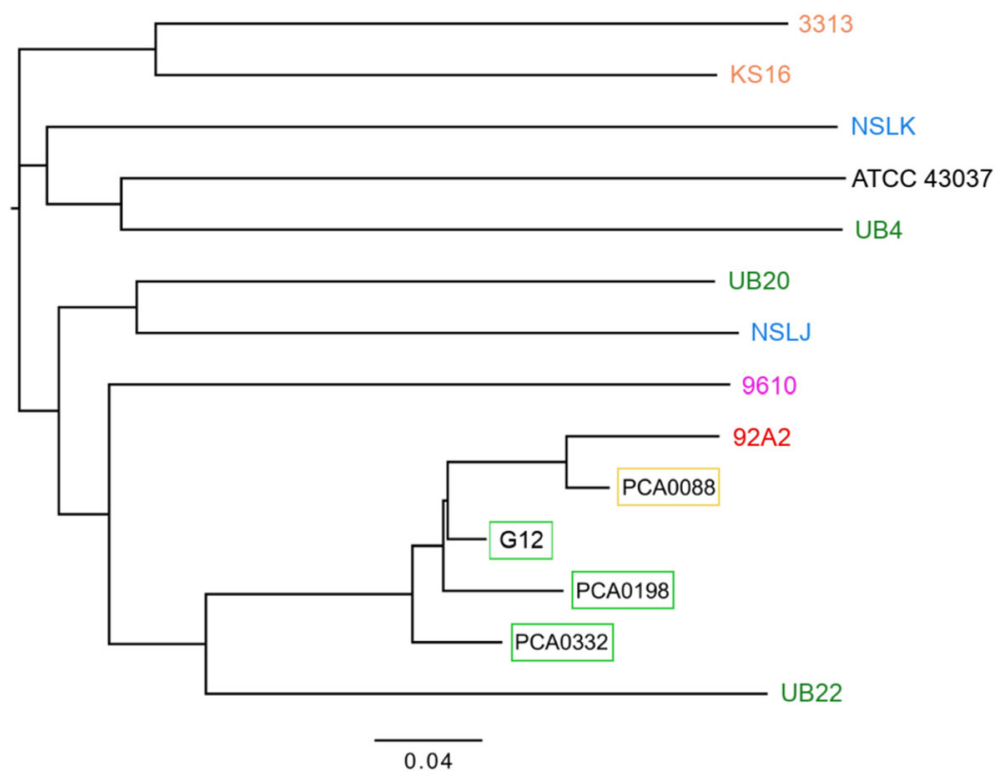

Fig. 2 The SNP phylogenetic tree showing the position of the Roman Iron Age (PCA0088) and medieval (PCA0198, PCA0332, and G12) T. forsythia genomes with respect to the genomes of modern $T$. forsythia identified worldwide

SNP tree (Fig. 2), the location of the G12 and PCA0198 genomes swapped. This effect might be caused by the fact that G12 had the shortest average read length, making more reads excluded in this sample (Supplementary Figure $4 \mathrm{~B}$ ), and by the previously mentioned properties of the polymerase used for G12 sequencing, which might lead to C/T and G/A SNP misidentification (Supplementary Figure $4 \mathrm{C}$ ). Moreover, the generation of SNP-based phylogenetic trees using 10 contemporary genomes and one ancient genome, again confirmed the latter one is always located next to the 92A2 genome (Supplementary Figure 4 D-G). Lastly, we repeated the computations using ATCC 43037 genome [41] as a reference. We identified 6541, 6360, 4711, 6635 SNPs for PCA0088, PCA0198, PCA0332 and G12 respectively (Supplementary Table 2 B). That is $\sim 4-5$ fold more than when using $92 \mathrm{~A} 2$ as a reference. This result is an obvious consequence of the phylogenetic relations among the studied T. forsythia isolates. Regardless of which genome was applied as a reference (92A2 or ATCC 43037) the ancient ones clustered with $92 \mathrm{~A} 2$ and 9610 genomes (Supplementary Figure 4 $\mathrm{H}$ ).

We also analyzed the sequence identity and deletions in T. forsythia genomes using CGView [42]. In comparison to the reference genome, the Roman Iron Age PCA0088 genome had 45 deletions (>500 nt, Supplementary Table 3 ), while in the medieval $T$. forsythia PCA0198, PCA0332 and G12 genomes, we identified 44, 54 and 60 deletions (>500 nt), respectively. As shown in Fig. 3, the deletions occurred within mainly coding regions and were detected both in contemporary and ancient $T$. forsythia genomes; $100,95.45,100$ and $75 \%$ of deletions (> $500 \mathrm{nt}$ ) identified in PCA0088, PCA0198, PCA0332 and G12, respectively, were also present in at least one contemporary genome. Additionally, $4.19 \%$ of deletions (> $500 \mathrm{nt}$ ) were present in all four ancient and in all nine contemporary genomes except for the reference genome. Among them, the largest deletion $(45,667 \mathrm{nt})$ was present in all of the genomes (except the reference genome) and carried tetracycline resistance genes. The high repeatability of deletions in the analyzed genomes is evidence that the absence of most regions in the ancient genomes might not be caused by random DNA degradation.

\section{T. forsythia virulence factors in ancient and contemporary strains}

To learn more about changes that occurred in the genetic structure of the $T$. forsythia population within the last 2 thousand years, we assessed variation in the genes that are known to be associated with the pathogenic activity of this bacterium and consequently are crucial for its survival. The analysis also included the $b s p B$ of unknown function because of its high sequence similarity to the well-known virulence factor, $b s p A$. The studied genes are listed in Supplementary Table $4 \mathrm{~A}$, and their genomic location in the 92A2 reference genome is presented in Fig. 3. For KLIKK proteases, we used our inhome sequenced KLIKK locus as a reference [10] since it was previously shown to be incorrectly assembled in the 92A2 genome [43]. To evaluate variation in the virulence factor genes, NGS reads used to reconstruct ancient and modern genomes were mapped to the reference genome. 


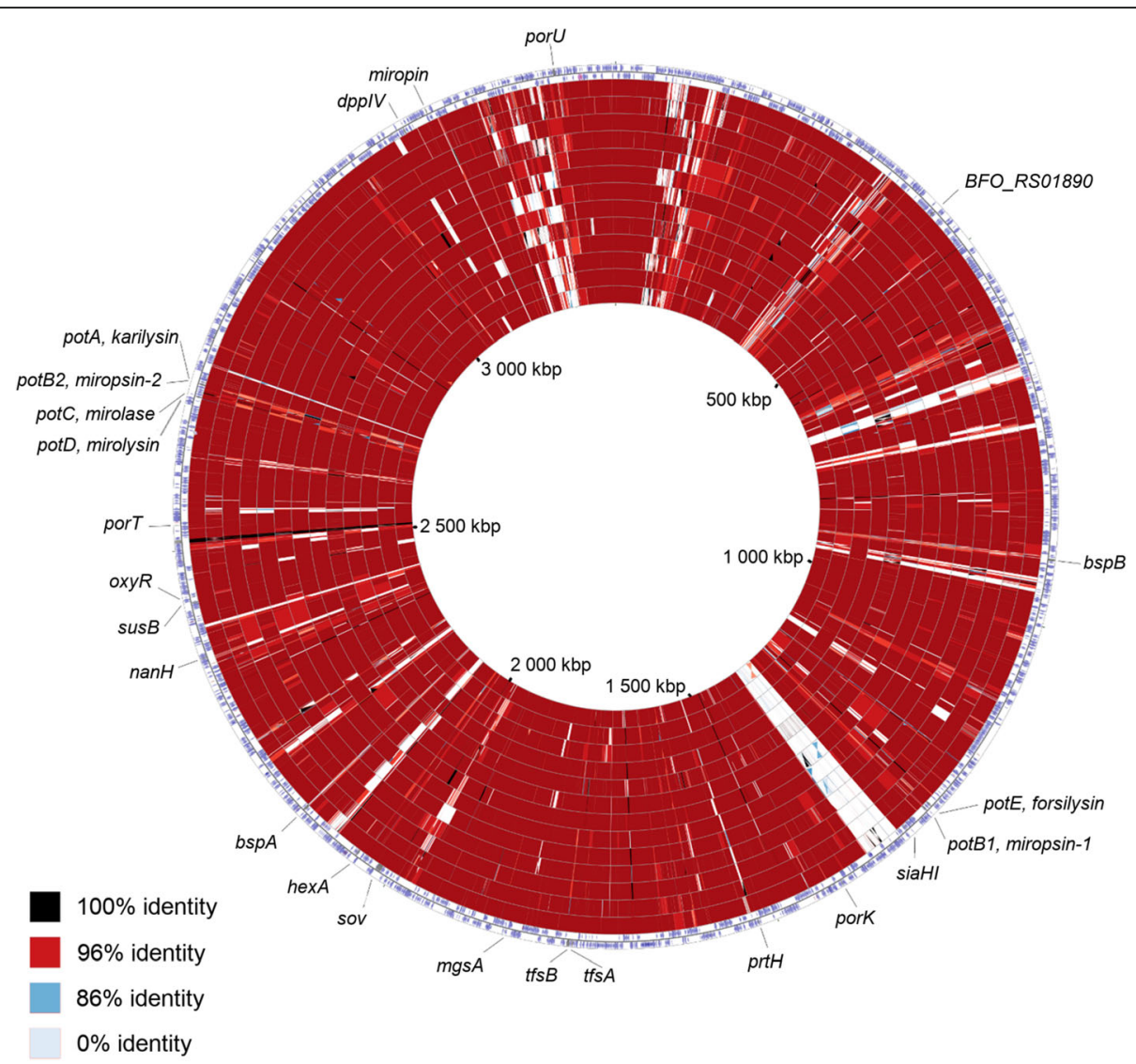

Fig. 3 DNA sequence comparison of the $T$. forsythia reference genome to the ancient $T$. forsythia genomes and to publicly available modern $T$. forsythia genomes. The two outermost rings depict the forward and reverse coding strands of the reference genome. The next 13 rings moving towards the inner part of the figure display regions of sequence similarity detected by BLAST comparison between the DNA of the reference genome and the DNA of the 13 compared T. forsythia genomes. The following genome order reflects the order of the circles starting from the outer part of the figure and moving towards the inner part: PCA0332, PCA0198, UB20, PCA0088, KS16, UB4, UB22, NSLJ, G12, 3313, NSLK, ATCC 43037, and 9610. Genes associated with T. forsythia virulence are labeled in the plot

As a result, we determined to what extent the virulence genes in the reference genome were covered by NGS reads. If gene coverage was almost complete $(>70 \%$ of nucleotides within the region occupied by the gene were covered by NGS reads), we inferred that the gene was present in the analyzed genome. If the gene coverage was moderate, most likely the analyzed genome did not possess this gene or the gene differed remarkably from the one present in the reference genome.

We found that KLIKK protease genes, together with associated upstream ORFs encoding small lipoproteins (pot), were the most variable group among the analyzed virulence factors (Fig. 4, Supplementary Table 4 A, B). Their average gene coverage in ancient genomes was $87.16 \%$ (from $17.44 \%$ potB2 in PCA0088 to $100 \%$ ). The average nucleotide coverage was 4.13 (PCA0088), 7.19 (PCA0198), 9.48 (PCA0332) and 8.33 (G12), which corresponds well to the average nucleotide coverage in the whole analyzed ancient genomes (Supplementary Table 1).
Miropsin-1, karilysin, mirolase, mirolysin and accompanying lipoprotein genes $(\operatorname{pot} B 1, \operatorname{pot} A, \operatorname{pot} C, \operatorname{pot} D)$ displayed high DNA sequence conservation both in ancient (average gene coverage: $94.64 \%$, from $66.52 \%$ in PCA0088 to 100\%) and in contemporary genomes (average gene coverage: $93.96 \%$, from $0 \%$ in NSLK to $100 \%$, Fig. 4). The only exceptions were the KS16 genome, which seems to be lacking potB1 (gene coverage: $15.46 \%)$ and the NSKL genome, in which gene coverage of potA was $0 \%$ and karilysin was $46.94 \%$, as well as potD at $26.85 \%$ and mirolysin at $46.49 \%$.

Forsilysin and accompanying potE were well conserved in PCA0332 and G12 (gene coverage: 99.46-100\%), but PCA0088 and PCA0198 had only partial coverage of these genes (35.22 and 64.99\%, respectively), which implies sequence dissimilarities to those of the reference. Notably, the contemporary 9610 genome seems to lack the two genes, potE and forsilysin, as their gene coverage was 10.91 and $11.02 \%$, respectively. Other contemporary strains, however, displayed very 

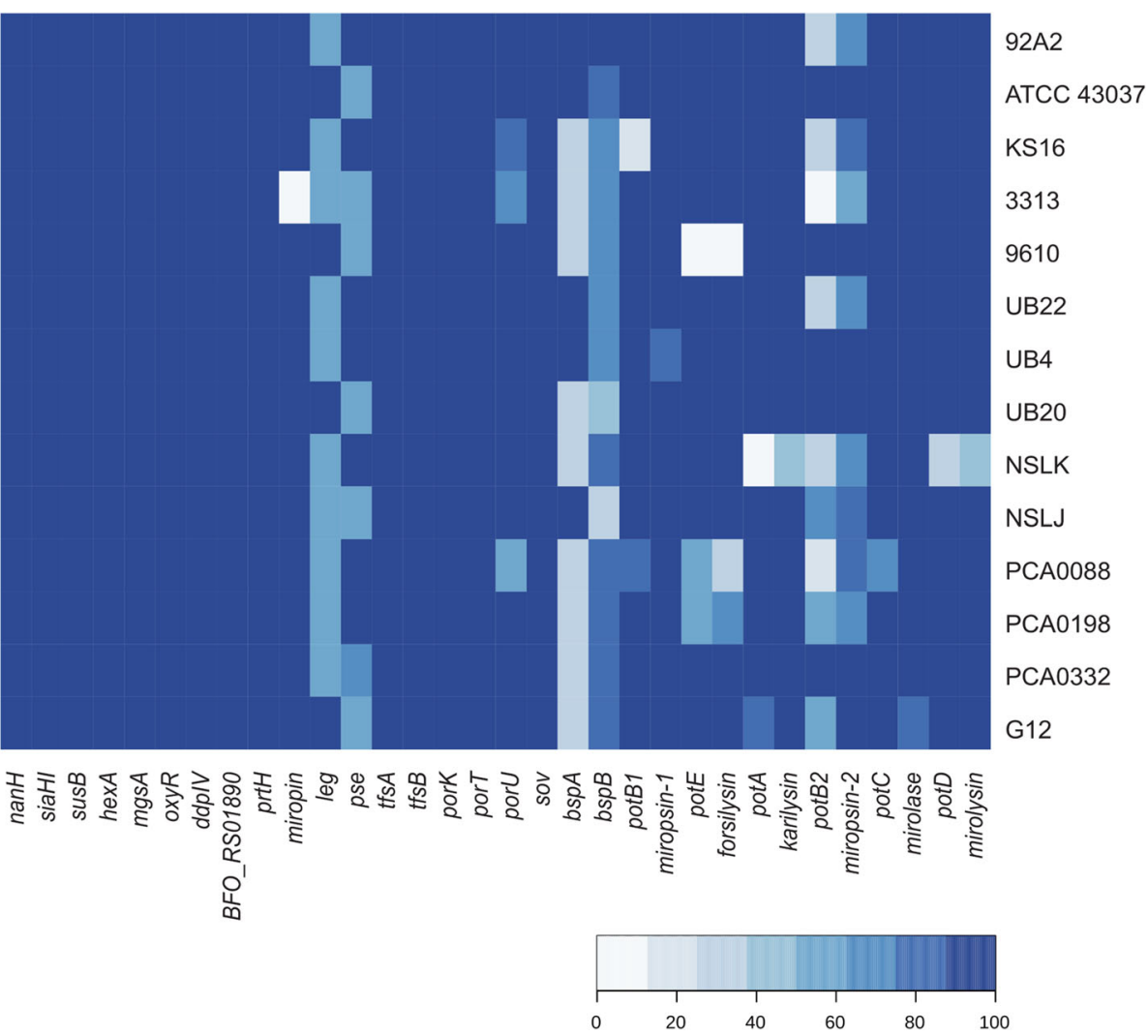

Fig. 4 The percentage sequence coverage of modern and ancient known virulence factor genes; the T. forsythia $92 A 2$ sequence was used as a reference, apart from leg when ATCC 43037 was used as a reference; and KLIKK protease genes when our in-home determined KLIKK protease locus was used as a reference [10]

high sequence conservation of these two genes $(100 \%$ gene coverage).

Miropsin-2 was well covered in all ancient genomes (from $71.38 \%$ in PCA0198 to $99 \%$ in PCA0332), but the accompanying potB2 was only partially covered in PCA0088 (17.44\%), PCA0198 (52.91\%) and G12 (52.13\%). Contemporary genomes displayed a similarly variable potB2 coverage pattern (average gene coverage: $59.60 \%$, from 0 to $100 \%$ ), which indicated high variability in this gene and even lack of this gene in extreme cases.

It is worth mentioning that in general, we discovered a high correlation between sequence coverage observed for operons composed of KLIKK protease genes directly preceded by genes encoding small lipoproteins referred to as Potempins with inhibitory activity directed specifically against the coexpressed protease (Potempa - data to be published) (Pearson correlation coefficient of 0.80). This finding might suggest a coevolution of those enzymes and their inhibitors.

Among other known T. forsythia virulence genes, only $b s p A$ (encoding the bacterial surface-associated protein) was not conserved in the ancient and most contemporary genomes (Fig. 5). Excluding bspA and pot-KLIKK, in the ancient genomes, the average gene coverage of genes

\begin{tabular}{|c|c|c|c|c|c|c|c|c|c|c|c|}
\hline & $92 A 2$ & $\begin{array}{l}\text { ATCC } \\
43037\end{array}$ & 9610 & 3313 & KS16 & KS16 & UB4 & UB20 & NSLJ & NSLJ & NSLK \\
\hline & \begin{tabular}{|c|} 
BFO_RS1 \\
4480 \\
\end{tabular} & \begin{tabular}{|c|} 
Tanf_RS1_R6 \\
3865
\end{tabular} & $\begin{array}{c}\text { BGK60_R } \\
S 08080\end{array}$ & $\begin{array}{c}\text { TF3313_R } \\
\text { S08530 }\end{array}$ & \begin{tabular}{|c|} 
TFKS16_R \\
$S 08260$ \\
\end{tabular} & $\begin{array}{c}\text { TFKS16_R } \\
\text { S08255 } \\
\end{array}$ & $\begin{array}{c}\text { BJU00 RS } \\
03515\end{array}$ & \begin{tabular}{|c|} 
BJT84RSS \\
04075 \\
\end{tabular} & \begin{tabular}{|c|} 
CLI86 113 \\
30
\end{tabular} & $\begin{array}{c}C L 186 \quad 135 \\
80\end{array}$ & $\begin{array}{c}\mathrm{CLI} 85 \quad 120 \\
20 \\
\end{array}$ \\
\hline PCA0088 & 19.42 & \begin{tabular}{|r|}
23.41 \\
\end{tabular} & 27.54 & 50.75 & \begin{tabular}{|r|}
43.94 \\
\end{tabular} & \begin{tabular}{|l|}
44.15 \\
\end{tabular} & $\begin{array}{r}28.31 \\
\end{array}$ & \begin{tabular}{|r|}
45.92 \\
\end{tabular} & \begin{tabular}{|r|}
32.64 \\
\end{tabular} & 23.75 & $\begin{array}{r}21.62 \\
\end{array}$ \\
\hline PCA0198 & 18.75 & 20.12 & 14.40 & 41.09 & 17.45 & 30.27 & 22.27 & 34.93 & 30.25 & 16.43 & 9.68 \\
\hline PCA0332 & 22.95 & 23.01 & 68.76 & 75.98 & 53.77 & 71.32 & 26.22 & 49.96 & 34.69 & 63.93 & 73.86 \\
\hline G12 & 15.00 & 21.10 & 77.54 & 72.03 & 67.66 & 55.01 & 22.61 & 53.01 & 31.95 & 61.89 & 73.81 \\
\hline
\end{tabular}

Fig. 5 The percentage sequence coverage of the bspA in ancient genomes; 11 modern bspA sequences were used as a reference (for details, see Methods) 
encoding virulence factors was $99.13 \%$. This result implies high evolutionary conservation of virulenceassociated genes. The average nucleotide coverage was 7.39 (G12) to 11.88 (PCA0198).

The bspA gene was covered in ancient genomes at only $31.3,29.07,37.01$ and $32.20 \%$ (in PCA0088, PCA0198, PCA0332, G12, respectively). Interestingly, within this gene, only the fragment encoding the Ig-like domain was covered (Supplementary Figure 5). Because a highly similar Ig-like coding domain is also present in the $b s p B$ gene, one can speculate that the observed effect is a result of the mapping procedure rather than a real presence of $b s p A$ in the ancient genome. The reads mapper most likely would not distinguish between the Iglike domain sequences present in $b s p A$ and $b s p B$. In accordance with such a presumption, the sequences encoding less conserved LRR domains of $b s p A$ were not covered in ancient genomes.

In contrast, the analysis of the $b s p B$ revealed high sequence conservation in the ancient genomes (in both Iglike and LRR regions). The average $b s p B$ coverage was $78.93 \%$ (from $75.45 \%$ in PCA0198 to $81.02 \%$ in PCA0088), with the average nucleotide coverage ranging from 7.94 (G12) to 35.56 (PCA0088). Interestingly, the average nucleotide coverage of $b s p B$ was $\sim 5$ times higher than the average nucleotide coverage of the PCA0088 genome (7.03) and $\sim 3$ times higher than the average nucleotide coverage of the PCA0198 genome (9.81) (Supplementary Table $4 \mathrm{~A}$ ). The above mentioned average $\operatorname{sspB}$ nucleotide coverage in PCA0088 and in PCA0198, as well as the fact that $b s p A$ was moderately covered not only in ancient but also in 5 modern genomes (KS16, 3313, 9610, UB20 and NSLK; on average $33.01 \%$, Fig. 5), indicated a general sequence variability of the $b s p A$ gene. This result inspired us to analyze all $b s p A$ sequences present in modern $T$. forsythia. Such an analysis would answer the question of whether $b s p A$ in the ancient genomes is more similar to one of the currently existing forms of $b s p A$ not present in the reference genome. In total, we identified 11 bsp $A$ complete sequences listed in Methods, " $b s p A$ analyses". Additionally, we identified $47 \mathrm{bspA}$ homologs and included them in the phylogenetic tree (Supplementary Figure 6). The $b s p A$ was identified in all of the modern genomes (UB22 had an incomplete gene at the contig boundary and was thus excluded from the analysis). Notably, some genomes seemed to have more than one copy of the bspAlike gene, particularly KS16 and NSLK.

Subsequently, we mapped reads of ancient NGS datasets to the retrieved 11 modern $b s p A$ sequences. The results are presented in Fig. 5. The coverage of the $b s p A$ in samples PCA0088 and PCA0198 did not exceed 50\%, regardless of the modern reference used (PCA0088 mapped best to the $b s p A$ sequence from UB20, 45.92\%;
PCA0198 to the $b s p A$ from 3313, 41.09\%). PCA0332 achieved $75.98 \%$ bspA coverage ( $b s p A$ sequence from 3313). Mapping of G12 also resulted in very high $b s p A$ coverage (max. $77.54 \%$ in 9610 ).

\section{Discussion}

We showed previously that the study of ancient bacterial species in human archaeological samples is possible [28]. In this work, we not only identified $T$. forsythia aDNA in teeth from Roman Iron Age and medieval human skeletons but also attempted complete genome assembly and sequence diversity analyses, paying special attention to virulence-related genes.

The NGS datasets were generated by shotgun sequencing without application of an enrichment procedure, as previously reported for other ancient bacteria [44, 45]. In total, 344 NGS datasets were scanned to trace the presence of $T$. forsythia. Three samples (Roman Iron Age PCA0088 and medieval PCA0198 and PCA0332) contained a sufficient amount of aDNA of this oral pathogen for a genome assembly. Additionally, we included in the analyses previously published NGS data on a $T$. forsythia genome recovered from a medieval skeleton found in Germany [30], as this genome was not previously analyzed in the phylogenetic/virulence gene context. The length distribution of DNA fragments and the damage patterns observed for T. forsythia DNA found in the four ancient samples showed damage characteristics typical of aDNA.

Anthropological examination of the four skulls from which aDNA was sampled uncovered alveolar bone loss in each of them, which indicated advanced periodontitis. In keeping with the present-day microbiological etiology of periodontitis, metagenomics analysis showed that in addition to $T$. forsythia, other disease-associated oral bacteria were present in the samples. We identified the "red complex" members ( $P$. gingivalis and $T$. denticola) in all four samples. In addition, F. alocis, Lachnospiraceae oral taxon 107, T. medium, T. vincentii, P. intermedia, and G. elegans were found in at least one of the samples. Importantly, the infection-associated species were significantly more abundant in the four selected samples than in 17 ancient reference samples. In particular, statistically significant changes in the amount of bacterial DNA were found for the following species: $T$. medium, T. vincentii (t-test, $p$-val $<0.001), P$. intermedia, $P$. gingivalis (t-test, $p$-val $<0.01), T$. forsythia, and G. elegans ( $\mathrm{t}$-test, $p$-val <0.05). Taken together, these data showed strong evidence that periodontal problems existed during the Roman Iron Age and medieval times, as they do now, despites different dietary, hygienic and lifestyle habits. The likelihood that the reference set contained samples derived from periodontitis sites only strengthen this general conclusion that the same 
pathogenic species were responsible for development of periodontitis today and thousands of years ago.

The SNP phylogenetic tree of modern T. forsythia fits the epidemiological and geographical contexts, confirming that $T$. forsythia is commonly present in different oral infections worldwide. Only the "Japanese branch" can be easily distinguished (KS16 and 3313). We hypothesize that this might be a consequence of population's isolation (on the island) or specific dietary habits. The location of other genomes (isolated in Europe and in the USA) on the phylogenetic tree does not always correlate with their geographical origin. This result indicates a worldwide diversity of this oral bacterium. On the other hand, the close location of the ancient genomes on the phylogenetic tree indicates a lower diversity of $T$. forsythia in the Roman Iron Age and medieval central Europe than currently exists.

Analyses of virulence factor genes revealed huge sequence variability in genes encoding the BspA protein and Potempins/KLIKK proteases, as it was shown recently by Zwickl et al. [41]. In all bacterial strains studied except two, 3313 (miropsin-2) and NSKL (karilysin), KLIKK proteases were directly preceded by ORFs encoding an inhibitor (potempin) specific for a downstream protease (Potempa et al., manuscript in preparation). Generally, regarding the potB2/miropsin pair, there were more sequence variations in the protease genes than in the accompanying inhibitor genes. Specifically, our findings indicate that potE, forsilysin, and potB2 sequences in PCA0088 and PCA0198, as well as potB2 in G12, might differ from those present in the modern reference sequences (KP715368 and KP715369). At the same time, potE and forsilysin were well conserved in all modern genomes except one, 9610, in which these genes were covered at only 10.91 and $11.02 \%$, respectively. This result might be caused, however, by the 9610 genome incompleteness (the genome is at the scaffold level). The obtained results suggest that other unknown forms of these genes existed in the past or even exist today. The analysis of potB2 and miropsin-2 coverage in modern genomes suggests general worldwide sequence diversity. This fact is most likely reflected by the difference in protease specificity, to which inhibitor specificity had to be adjusted. In this respect, the observed variation may illustrate the evolution of inhibitors to match the changing specificity of proteases. This fact supports the thesis that a generally high Pearson correlation coefficient $(0.80)$ was observed between the KLIKK enzyme and the corresponding POT inhibitor sequence coverage in different $T$. forsythia genomes.

Analysis of $b s p A$ and $b s p B$ revealed high sequence diversity of these genes among modern and ancient genomes. This may be related to the tandem occurrence of immunoglobulin (Ig) protein domains in BspA and
BspB. The Ig-like domains share the tertiary structure despite very low conservation of the amino acid sequence. In prokaryotes they are often found on the cell surface responsible for host adhesion and presentation of ligand binding domains [46]. We speculate that $b s p A$ diversity is an adaptation of different strains of $T$. forsythia to specific microflora. PCA0332 and G12 had bspA sequences most similar to those presented in the modern genomes 3313 and 9610, respectively. Our findings also suggest that the Roman Iron Age and medieval T. forsythia (PCA0088 and PCA0198) might lack ancient or contemporary forms of $b s p A$. The deletion analysis further supports our claim that the absence of $b s p A$ cannot be explained by random DNA degradation. As several important functions for bacterial survival have been shown for the BspA protein [6], it seems possible that an unknown homolog exerted BspA functions or that at least some of them exist in PCA0332 and in G12. This hypothesis is supported by the observation that nucleotide coverage of the $b s p B$ in these genomes is higher than the average coverage (that for PCA0088 was 35.56 and for PCA0198 was 30.56, whereas average nucleotide coverage was 7.03 and 9.81, respectively). $b s p B$ displays very high sequence similarity to $b s p A$, but the former protein has not yet been investigated for its function(s). This finding may indicate that in the absence of the typical $b s p A$, the mapper assigned reads to the most similar sequence in the $92 \mathrm{~A} 2$ reference genome, i.e., to $b s p B$. This finding suggests that another homolog (or homologs) of the $b s p A$ containing LRRs and Ig-like motifs might be present in the ancient genomes. Alternatively, PCA0088 and PCA0198 might lack a functional form of BspA, or the BspA LRR domains with diverged sequences adopted functions different than those in other T. forsythia strains. To answer this intriguing question longer reads (e.g. from Nanopore sequencing) might be helpful, however, only if the genomic fragment containing the $b s p A$ sequence was well preserved.

In contrast to sequence variations in $\mathrm{BspA} / \mathrm{BspB}$, TfsA/TfsB and Potempins/KLIKK proteases, other virulence factors involved in carbohydrate degradation and using sialic acid as a carbon source, DPPIV, OxyR, and components of the T9SS, are nearly identical in all $T$. forsythia strains. Considering very close relationship of T. forsythia with the human host the strict conservation of the sialic acid catabolism and transport operon apparently represents a human-specific adaptation and it is plausible that $T$. forsythia co-evolved with humans [47]. Also, the biosynthesis pathways of O-glycans decorating surface proteins, including TfsA and TfsB, with nonulosonic acid are nearly identical in the ancient and modern strains of T. forsythia. However, while PCA088 and PCA0198 shared the pse gene cluster present in ATCC 43037 and UB20, G12 had the $\operatorname{leg}$ genes as found in 
92A2, UB4, KS16 and UB22. This indicates that none of these two nonulosonic acid variants provided $T$. forsythia with the cutting-edge adaptive advantage to coexist with humans as a member of the complex dental plaque microbiome. Considering differences in host response to $T$. forsythia expressing different variants of nonulosonic acid [48], it will be interesting to see their association with the periodontal health, other periodontal pathogens and progression of periodontitis.

Among tightly conserved proteins, Miropin deserves special emphasis. As a protease inhibitor of the serpin superfamily, it possesses an exposed reactive center loop (RCL) with the residue at the $\mathrm{P} 1$ position and a sequence upstream of the P1-P1' reactive site peptide bond attacked by the targeted protease that dictates the serpin's inhibitory specificity. Using the conserved serpin scaffold, eukaryotic multicellular organisms evolved a multitude of serpins with discrete specificity dependent on variations in the RCL sequence [49]. In this context, it is very interesting to note that not only the serpin scaffold is conserved but also the RCL is identical across ancient and present-day strains of $T$. forsythia. Notably, variations in the miropin sequence in PCA0088 are outside the RCL. This pattern is in stark contrast to that for two recently recognized oral taxa of Tannerella spp. [49], which encode at least 12 serpins varying within the RCL and thus exerting different inhibitory specificities (our unpublished analysis). Of note, these strains never cluster with $T$. forsythia and may represent new species. Taken together, these data indicate that the conservation of miropin's RCL through millennia suggests an essential role for this protein in T. forsythia "well-being" in the crowded environment of the subgingival biofilm together with highly proteolytic $P$. gingivalis and exposure to host proteases.

\section{Conclusion}

T. forsythia genomes manifest high sequence similarity, with the indication that the modern worldwide genome diversity seems to be higher than that observed in the first millennium AD in Europe. Sequencing of more ancient $T$. forsythia genomes will verify this assumption. Furthermore, we showed that some virulence-associated genes (several pot/KLIKK and $b s p A$ ) vary significantly between both modern and ancient T. forsythia. As an extreme example, the 9610 genome seems to be lacking forsylisin, while PCA0088 and PCA0198 most likely had more than 2 copies of a $b s p A$-like of unknown sequence. This fact, together with the observed periodontitischaracteristic alveolar bone lesions in the mandible and maxilla of ancient skeletons, suggests that variations in the $b s p A / P o t-K L I K K$ loci did not affect the virulence of the ancient dysbiotic biofilm. Finally, we observed that the sequences of $b s p A$ vary significantly among modern genomes. These findings are important for further $T$. forsythia evolution studies, especially in the context of its virulence factors and adaptation to the host organism as a result of changing diet and hygienic habits.

\section{Methods}

\section{Sample source}

The biological material came from archaeological sites distributed across Poland. In total we examined 344 ancient human teeth, including 161 Roman Iron Age and medieval individuals from our previous studies, detailed description is available in $[28,50,51]$ and 183 medieval individuals from Ląd [37], Obłaczkowo [10], Brzeg [2], Gołuń [6], Poznań-Śródka [12], Opole [41], Końskie [9], Płońsk [20], Ostrów Lednicki [20], Dziekanowice [21], and Warszawa [5] (data to be published).

\section{Experimental procedures}

DNA was extracted from teeth in a sterile laboratory dedicated exclusively to ancient DNA (aDNA) study at the Adam Mickiewicz University in Poznan. In this laboratory, analyses on Tannerella cultures have never been carried out. During every step of aDNA analyses, all precautions against modern DNA contamination were kept, as previously described in [28]. At every stage of our analyses, including DNA extraction, library construction and PCR amplification (12 cycles), we set up two blanks to control potential DNA contamination. The blank controls did not yield any contaminating DNA. DNA was purified using the MinElute kit (QIAGEN) according to Yang et al. [52] and Malmstrom et al. [53]. $20 \mu \mathrm{l}$ of DNA isolate was used to prepare a genomic library as described in Meyer and Kircher [54]. Sequencing of genomic libraries was performed with GAIIx and HiSeq 4000 (Illumina) following the standard $100 \mathrm{bp}$ pair-end sequencing protocol. The average library fragment was $253 \mathrm{nt}, 264 \mathrm{nt}$ and $239 \mathrm{nt}$ long for PCA0088, PCA0088 and PCA0332, respectively.

\section{Bioinformatics procedures}

Metagenomics analyses were conducted with the default settings with MetaPhlAn2 [29], software that identifies bacteria, archaea, viruses and protozoa based on the reads mapping to marker sequences and estimates their percentage abundance in a sample. The genus habitat, Gram stain type and respiratory type were assigned manually based on the characteristics of species identified in the sample within this genus. For graphical representation, KRONA software [55] was used.

\section{T. forsythia genome analyses}

All reads were adapter-trimmed and, quality $(Q \geq 20)$ and length $(\geq 20)$ filtered with AdapterRemoval [56]. The PCR duplicates (PCA0088 28.13\% of reads, PCA0198 
$16.27 \%$ of reads, PCA0332 $19.38 \%$ of reads and G12 $19.17 \%$ of reads) were removed with picard-tools MarkDuplicates. Overlapping pair-end reads were collapsed with AdapterRemoval (-collapse option). Obtained reads were mapped to the reference $T$. forsythia genome ( $\mathrm{NC}_{-}$ 016610.1) with bwa ver. 0.7.10 and aln -1 1000 -n 0.001 parameters following a protocol described in [57]. Subsequently, mapped files with collapsed, remaining pairend reads and singletons were merged into one file with samtools, samtools merge [58]. Before mapping, we additionally trimmed 3 nucleotides from the beginning and 3 nucleotides from the end of the reads [57]. This step ensured that age-related transitions $(\mathrm{C}>\mathrm{T}$ and $\mathrm{G}>\mathrm{A})$, which are mostly present in the last 3 bases, did not influence mapping quality and reduced SNP false positives rate. A genome coverage plot was generated with Qualimap v2.2.1 [59] based on the final mapping file (collapsed, pair-end reads and singletons). Read length distribution was assessed with PRINSEQ [60]. The consensus sequence was obtained with the command: "samtools mpileup -uf reference_genome.fna mapped.bam | bcftools view -s $<$ (echo mapped.bam 1) -cg - | vcfutils.pl vcf2fq". Samtools mpileup produces "pileup" textual format from an alignment file (mapped.bam). Subsequently the file was processed with bcftools view (options -cg) that outputted gVCF blocks of homozygous reference calls, with depth ranges specified and minimum allele count of sites. Finally, the consensus sequence was extracted using vcfutils.pl. The DNA damage pattern of reads (full length) that mapped to the T. forsythia reference genome was assessed with MapDamage2.0 [35] software with default settings.

SNP calling was performed with GATK tools [37]. Only SNPs that fulfilled the following criteria were used: (i) genotyping quality $Q>30$, (ii) $>3$ (or $>10$ ) reads supported the SNP site, and (iii) the proportion of reads supporting the SNP was $>90 \%$. If the same three criteria were fulfilled but for the reference nucleotide, we called the reference nucleotide. If neither a reference nor a SNP was called, we assigned " $n$ " as an indicator of missing data.

\section{Modern T. forsythia genotyping}

Ten publicly available $T$. forsythia modern genomes were at different levels of assembly (3 complete genomes, 5 scaffolds, 2 contigs). To make the genotyping possible and the genomic coordinates comparable with each other, we (i) generated reads of length 100 for each of the genomes, with a shift of $1 \mathrm{nt}$ using our in-home script, (ii) mapped (with bwa aln -1 1000 -n 0.001) the artificially generated reads against the reference genome (92A2), and (iii) and used on the mapping output (.bam) the same approach for $\mathrm{SNP} /$ reference nucleotide calling as for ancient samples (see above).
Deletions were identified based on consensus sequence analyses. Our in-home script was used to scan consensus sequences for missing blocks of DNA sequence (" $n$ "). The full list of identified deletions is in Supplementary Table 3. Comparison of the ancient and modern genomes with the reference 92A2 was performed with CGView [42].

SNP phylogenetic tree construction from modern genomes was conducted with FastTree [38] using neighbor-joining and generalized time-reversible models of nucleotide evolution. Pplacer software [39] with the default settings was used to project the ancient stains (PCA0088, PCA0198, PCA0332, and G12) on the generated tree.

\section{BspA analyses}

The sequences of all BspA genes were retrieved from all T. forsythia publicly available modern genomes. We used the BLAST search tool blastn [61] and searched against the known $b s p A$ sequence identified in $T$. forsythia ATCC 43037. In this way, we identified the following functional bspA genes: BFO_RS14480 (92A2), Tanf_ RS13865 (ATCC 43037), BGK60_RS08080 (9610), TF3313_RS08530 (3313), TFKS16_RS08260 (KS16), TFKS16_RS08255 (KS16), BJU00_RS03515 (UB4), BJT84_RS04075 (UB20), CLI86_11330 (NSLJ), CLI86_ 13580 (NSLJ) and CLI85_12020 (NSLK).

\section{Supplementary information}

Supplementary information accompanies this paper at https://doi.org/10. 1186/s12864-020-06810-9.

Additional file 1. Supplementaty Tables.

Additional file 2. Supplementary Material "Anthropological description of analyzed individuals" and Supplementary Figures.

\section{Abbreviations}

aDNA: Ancient DNA; NGS: Next Generation Sequencing; LRR: Leucine-rich repeat; Ig-like: Immunoglobulin-like; SNP: Single nucleotide polymorphism; Ig: Immunoglobulin; RCL: Reactive center loop

\section{Acknowledgements}

The authors thank P. Kozłowski for inspirational discussions. We also thank K. Książkiewicz for his help with the preparation of Supplementary Figure $2 \mathrm{~A}$.

\section{Authors' contributions}

AP conceived the study, designed and coordinated the study, analyzed the data, discussed the results, wrote the manuscript and prepared figures; IS participated in the genome reconstructions and SNP and deletion analyses; LH prepared NGS libraries and ran NGS experiments; KN participated in SNP analyses and helped with figures preparation; AJ extracted DNA, participated in NGS library preparation, and participated in the anthropological examination of the skeletons; DT, WN and AW anthropological analyzed skeletons, interpreted data and described the results; JP analyzed and discussed the data, helped with the manuscript preparation; MF conceived the overall idea of the study, participated in the study design, analyzed and discussed the data, and was responsible for the final version of the manuscript; all authors read and approved the final manuscript. 


\section{Funding}

This work was supported by the Polish National Science Center [2014/12/W/ NZ2/00466 to MF. JP was supported by 2016/21/B/NZ1/00292 and NIH/ NIDCR R21DE026280.

\section{Availability of data and materials}

The datasets supporting the conclusions of this article are available in the National Center for Biotechnology Information (NCBI) Sequence Read Archive (SRA) repository under accession number: SRP093814 (https://trace. ncbi.nlm.nih.gov/Traces/sra/?study=SRP093814), and from the corresponding author on reasonable request. Data for G12 sample was downloaded from SRA (SRP029257, https://trace.ncbi.nlm.nih.gov/Traces/sra/?study=SRP029257). T. forsythia modern genomes were downloaded from NCBI Genome repository, 92A2 (assembly ID: GCA_000238215.1, https://www.ncbi.nlm.nih. gov/genome/11045?genome_assembly_id=231734), ATCC 43037 (assembly ID: GCA_006385365.1, https://www.ncbi.nlm.nih.gov/genome/11045 ?genome_assembly_id=590153), 9610 (assembly ID: GCA_001938785.1, https://www.ncbi.n/m.nih.gov/genome/11045?genome_assembly id=300815 ), 3313 (assembly ID: GCA_001547875.1, https://www.ncbi.nlm.nih.gov/ genome/11045?genome_assembly_id=264125), KS16 (assembly ID: GCA_001547855.1, https://www.ncbi.nlm.nih.gov/genome/11045?genome_ assembly_id=264124), UB4 (assembly ID: GCA_900096725.1, https://www. ncbi.nlm.nih.gov/genome/11045?genome_assembly_id=284387), UB20 (assembly ID: GCA_900096735.1, https://www.ncbi.nlm.nih.gov/genome/11 045?genome_assembly_id=284388), UB22 (assembly ID: GCA_900096715.1, https://www.ncbi.nlm.nih.gov/genome/11045?genome_assembly_id=284386 ), NSLJ (assembly ID: GCA_002529085.1, https://www.ncbi.nlm.nih.gov/ genome/11045?genome_assembly_id=340904), NSLK (assembly ID: GCA_002529295.1, https://www.ncbi.nlm.nih.gov/genome/11045?genome assembly_id=340905). bspA sequence of T. forsythia ATCC 43037 was obtained from NCBI Gene repository (locus tag: BFO_RS14480, https://www. ncbi.nlm.nih.gov/gene/34760141). The bspA sequences for the remaining modern T. forsythia were obtained by blastn search of their genomes (against BFO_RS14480). As a result we identified the following bspA-like genes BFO_RS14480 (92A2), Tanf_RS13865 (ATCC 43037), BGK60_RS08080 (9610), TF3313_RS08530 (3313), TFKS16_RS08260 (KS16), TFKS16_RS08255 (KS16), BJU00_RS03515 (UB4), BJT84_RS04075 (UB20), CLI86_11330 (NSLJ), CLI86_13580 (NSLJ) and CLI85_12020 (NSLK) which are available in NCBI Nucleotide repository. KLIKK locus sequence was obtained from NCBI Nucleotide repository (accession IDs: KP715368 https://www.ncbi.nlm.nih. gov/nuccore/KP715368 and KP715369 https://www.ncbi.nlm.nih.gov/ nuccore/KP715369).

\section{Ethics approval and consent to participate}

All 344 samples (human teeth) used in these studies for T. forsythia screening have been comprehensively analyzed within the NSC grant 2014/12/W/NZ2/ 00466 to MF. They came from 18 osteological collections, in each case adequate permission was obtained for the use of samples in genomic studies. In this work, we analyzed in details 3 samples. The disposers of the 3 samples (and of the skeletal remains the samples came from) co-authored this work.

\section{Consent for publication}

Not applicable.

\section{Competing interests}

The authors declare that they have no competing interests.

\section{Author details}

${ }^{1}$ Institute of Bioorganic Chemistry, Polish Academy of Sciences, 61-704 Poznan, Poland. ${ }^{2}$ Department of Human Evolutionary Biology, Institute of Anthropology, Faculty of Biology, Adam Mickiewicz University in Poznan, 61-614 Poznan, Poland. ${ }^{3}$ Department of Human Biology, Faculty of Biological Sciences, Wroclaw University, 50-138 Wroclaw, Poland. ${ }^{4}$ Anthropological Laboratory, Museum of the First Piasts at Lednica, 62-261 Lednogora, Poland. ${ }^{5}$ Faculty of Biochemistry, Biophysics and Biotechnology, Jagiellonian University, 30-387 Krakow, Poland. 'Department of Oral Immunity and Infectious Diseases, University of Louisville School of Dentistry, Louisville, KY 40202, USA. ${ }^{7}$ Institute of Computing Science, Poznan University of Technology, 60-965 Poznan, Poland.
Received: 12 February 2020 Accepted: 8 June 2020

Published online: 15 June 2020

\section{References}

1. Eke PI, Dye BA, Wei L, Slade GD, Thornton-Evans GO, Borgnakke WS, et al. Update on prevalence of periodontitis in adults in the United States: NHANES 2009 to 2012. J Periodontol. 2015;86(5):611-22.

2. Hajishengallis G. Periodontitis: from microbial immune subversion to systemic inflammation. Nat Rev Immunol. 2015;15(1):30-44.

3. Darveau RP. Periodontitis: a polymicrobial disruption of host homeostasis. Nat Rev Microbiol. 2010;8(7):481-90.

4. Endo A, Watanabe T, Ogata N, Nozawa T, Aikawa C, Arakawa S, et al. Comparative genome analysis and identification of competitive and cooperative interactions in a polymicrobial disease. ISME J. 2015;9(3):629-42.

5. Lamont RJ, Hajishengallis G. Polymicrobial synergy and dysbiosis in inflammatory disease. Trends Mol Med. 2015;21(3):172-83.

6. Sharma A. Virulence mechanisms of Tannerella forsythia. Periodontology 2000. 2010;54(1):106-16.

7. Lourenco TG, Heller D, Silva-Boghossian CM, Cotton SL, Paster BJ, Colombo AP. Microbial signature profiles of periodontally healthy and diseased patients. J Clin Periodontol. 2014:41(11):1027-36.

8. Chen H, Liu Y, Zhang M, Wang G, Qi Z, Bridgewater L, et al. A Filifactor alocis-centered co-occurrence group associates with periodontitis across different oral habitats. Sci Rep. 2015;5:9053.

9. Saito T, Ishihara K, Kato T, Okuda K. Cloning, expression, and sequencing of a protease gene from Bacteroides forsythus ATCC 43037 in Escherichia coli. Infect Immun. 1997;65(11):4888-91.

10. Ksiazek M, Mizgalska D, Eick S, Thogersen IB, Enghild JJ, Potempa J. KLIKK proteases of Tannerella forsythia: putative virulence factors with a unique domain structure. Front Microbiol. 2015;6:312.

11. Koziel J, Karim AY, Przybyszewska K, Ksiazek M, Rapala-Kozik M, Nguyen KA, et al. Proteolytic inactivation of LL-37 by karilysin, a novel virulence mechanism of Tannerella forsythia. J Innate Immun. 2010;2(3):288-93.

12. Jusko M, Potempa J, Karim AY, Ksiazek M, Riesbeck K, Garred P, et al. A metalloproteinase karilysin present in the majority of Tannerella forsythia isolates inhibits all pathways of the complement system. J Immunol. 2012; 188(5):2338-49.

13. Jusko M, Potempa J, Mizgalska D, Bielecka E, Ksiazek M, Riesbeck K, et al. A metalloproteinase Mirolysin of Tannerella forsythia inhibits all pathways of the complement system. J Immunol. 2015;195(5):2231-40.

14. Kumagai $Y$, Yagishita H, Yajima A, Okamoto T, Konishi K. Molecular mechanism for connective tissue destruction by dipeptidyl aminopeptidase IV produced by the periodontal pathogen Porphyromonas gingivalis. Infect Immun. 2005;73(5):2655-64.

15. Yost S, Duran-Pinedo AE. The contribution of Tannerella forsythia dipeptidyl aminopeptidase IV in the breakdown of collagen. Mol Oral Microbiol. 2018; 33(6):407-19. https://doi.org/10.1111/omi.12244.

16. Ksiazek M, Mizgalska D, Enghild JJ, Scavenius C, Thogersen IB, Potempa J. Miropin, a novel bacterial serpin from the periodontopathogen Tannerella forsythia, inhibits a broad range of proteases by using different peptide bonds within the reactive center loop. J Biol Chem. 2015;290(1):658-70.

17. Ishikura H, Arakawa S, Nakajima T, Tsuchida N, Ishikawa I. Cloning of the Tannerella forsythensis (Bacteroides forsythus) siaHl gene and purification of the sialidase enzyme. J Med Microbiol. 2003;52(Pt 12):1101-7.

18. Thompson H, Homer KA, Rao S, Booth V, Hosie AH. An orthologue of Bacteroides fragilis $\mathrm{NanH}$ is the principal sialidase in Tannerella forsythia. J Bacteriol. 2009;191(11):3623-8.

19. Honma K, Mishima E, Sharma A. Role of Tannerella forsythia NanH sialidase in epithelial cell attachment. Infect Immun. 2011;79(1):393-401.

20. Hughes CV, Malki G, Loo CY, Tanner AC, Ganeshkumar N. Cloning and expression of alpha-D-glucosidase and $\mathrm{N}$-acetyl-beta-glucosaminidase from the periodontal pathogen, Tannerella forsythensis (Bacteroides forsythus). Oral Microbiol Immunol. 2003;18(5):309-12.

21. Honma K, Mishima E, Inagaki S, Sharma A. The OxyR homologue in Tannerella forsythia regulates expression of oxidative stress responses and biofilm formation. Microbiology. 2009;155(Pt 6):1912-22.

22. Lasica AM, Ksiazek M, Madej M, Potempa J. The type IX secretion system (T9SS): highlights and recent insights into its structure and function. Front Cell Infect Microbiol. 2017;7:215. 
23. Honma K, Inagaki S, Okuda K, Kuramitsu HK, Sharma A. Role of a Tannerella forsythia exopolysaccharide synthesis operon in biofilm development. Microb Pathog. 2007:42(4):156-66.

24. Narita Y, Sato K, Yukitake H, Shoji M, Nakane D, Nagano K, et al. Lack of a surface layer in Tannerella forsythia mutants deficient in the type IX secretion system. Microbiology. 2014;160(Pt 10):2295-303.

25. Sharma A, Sojar HT, Glurich I, Honma K, Kuramitsu HK, Genco RJ. Cloning, expression, and sequencing of a cell surface antigen containing a leucinerich repeat motif from Bacteroides forsythus ATCC 43037. Infect Immun. 1998;66(12):5703-10

26. Ikegami A, Honma K, Sharma A, Kuramitsu HK. Multiple functions of the leucine-rich repeat protein LrrA of Treponema denticola. Infect Immun. 2004;72(8):4619-27.

27. Veith PD, O'Brien-Simpson NM, Tan Y, Djatmiko DC, Dashper SG, Reynolds EC. Outer membrane proteome and antigens of Tannerella forsythia. J Proteome Res. 2009;8(9):4279-92.

28. Philips A, Stolarek I, Kuczkowska B, Juras A, Handschuh L, Piontek J, et al. Comprehensive analysis of microorganisms accompanying human archaeological remains. GigaScience. 2017;6(7):1-13.

29. Truong DT, Franzosa EA, Tickle TL, Scholz M, Weingart G, Pasolli E, et al. MetaPhIAn2 for enhanced metagenomic taxonomic profiling. Nat Methods. 2015;12(10):902-3.

30. Warinner C, Rodrigues JF, Vyas R, Trachsel C, Shved N, Grossmann J, et al. Pathogens and host immunity in the ancient human oral cavity. Nat Genet. 2014:46(4):336-44.

31. Griffen AL, Beall CJ, Campbell JH, Firestone ND, Kumar PS, Yang ZK, et al. Distinct and complex bacterial profiles in human periodontitis and health revealed by 165 pyrosequencing. ISME J. 2012;6(6):1176-85

32. Kokowski A. Grupa masłomęcka i jej cmentarzyska w: Starożytna Polska. Od trzeciego wieku przed Chrystusem do starożytności. Wydawnictwo TRIO. 2006; 1:385-412

33. Kokowski A. Archeologia Gotów. Goci z Kotliny Hrubieszowskiej. Idea Media. 1999. ISBN: 8390734176.

34. Krzyżaniak L, Muzeum A. Wczesnośredniowieczne cmentarzyska szkieletowe w Ladzie, woj. Konin. Poznan: Muzeum Archeologiczne w Poznaniu; 1986.

35. Jonsson $\mathrm{H}$, Ginolhac A, Schubert $M$, Johnson PL, Orlando L. mapDamage2.0: fast approximate Bayesian estimates of ancient DNA damage parameters. Bioinformatics. 2013;29(13):1682-4.

36. Rasmussen M, Li Y, Lindgreen S, Pedersen JS, Albrechtsen A, Moltke I, et al. Ancient human genome sequence of an extinct Palaeo-Eskimo. Nature. 2010;463(7282):757-62

37. DePristo MA, Banks E, Poplin R, Garimella KV, Maguire JR, Hartl C, et al. A framework for variation discovery and genotyping using next-generation DNA sequencing data. Nat Genet. 2011;43(5):491-8.

38. Price MN, Dehal PS, Arkin AP. FastTree: computing large minimum evolution trees with profiles instead of a distance matrix. Mol Biol Evol. 2009;26(7):1641-50.

39. Matsen FA, Kodner RB, Armbrust EV. pplacer: linear time maximumlikelihood and Bayesian phylogenetic placement of sequences onto a fixed reference tree. BMC Bioinformatics. 2010;11:538.

40. Green RE, Briggs AW, Krause J, Prufer K, Burbano HA, Siebauer M, et al. The Neandertal genome and ancient DNA authenticity. EMBO J. 2009;28(17):2494-502.

41. Zwickl NF, Stralis-Pavese N, Schaffer C, Dohm JC, Himmelbauer H. Comparative genome characterization of the periodontal pathogen Tannerella forsythia. BMC Genomics. 2020;21(1):150.

42. Stothard $P$, Wishart DS. Circular genome visualization and exploration using CGView. Bioinformatics. 2005;21(4):537-9.

43. Karim AY, Kulczycka M, Kantyka T, Dubin G, Jabaiah A, Daugherty PS, et al. A novel matrix metalloprotease-like enzyme (karilysin) of the periodontal pathogen Tannerella forsythia ATCC 43037. Biol Chem. 2010;391(1):105-17.

44. Schuenemann VJ, Singh P, Mendum TA, Krause-Kyora B, Jager G, Bos KI, et al. Genome-wide comparison of medieval and modern Mycobacterium leprae. Science. 2013;341(6142):179-83.

45. Maixner F, Krause-Kyora B, Turaev D, Herbig A, Hoopmann MR, Hallows JL, et al. The 5300-year-old helicobacter pylori genome of the iceman. Science. 2016;351(6269):162-5.

46. Bodelon G, Palomino C, Fernandez LA. Immunoglobulin domains in Escherichia coli and other enterobacteria: from pathogenesis to applications in antibody technologies. FEMS Microbiol Rev. 2013;37(2):204-50.

47. Stafford G, Roy S, Honma K, Sharma A. Sialic acid, periodontal pathogens and Tannerella forsythia: stick around and enjoy the feast! Mol Oral Microbiol. 2012;27(1):11-22.
48. Bloch S, Tomek MB, Friedrich V, Messner P, Schaffer C. Nonulosonic acids contribute to the pathogenicity of the oral bacterium Tannerella forsythia. Interface Focus. 2019;9(2):20180064.

49. Silverman GA, Bird PI, Carrell RW, Church FC, Coughlin PB, Gettins PG, et al. The serpins are an expanding superfamily of structurally similar but functionally diverse proteins. Evolution, mechanism of inhibition, novel functions, and a revised nomenclature. J Biol Chem. 2001;276(36):33293-6.

50. Stolarek I, Juras A, Handschuh L, Marcinkowska-Swojak M, Philips A, Zenczak $M$, et al. A mosaic genetic structure of the human population living in the South Baltic region during the Iron age. Sci Rep. 2018;8(1):2455.

51. Stolarek I, Handschuh L, Juras A, Nowaczewska W, Kocka-Krenz H, Michalowski A, et al. Goth migration induced changes in the matrilineal genetic structure of the central-east European population. Sci Rep. 2019;9(1):6737.

52. Yang DY, Eng B, Waye JS, Dudar JC, Saunders SR. Technical note: improved DNA extraction from ancient bones using silica-based spin columns. Am J Phys Anthropol. 1998;105(4):539-43.

53. Malmstrom H, Svensson EM, Gilbert MT, Willerslev E, Gotherstrom A, Holmlund G. More on contamination: the use of asymmetric molecular behavior to identify authentic ancient human DNA. Mol Biol Evol. 2007; 24(4):998-1004.

54. Meyer M, Kircher M. Illumina sequencing library preparation for highly multiplexed target capture and sequencing. Cold Spring Harbor Protocols. 2010;2010(6):pdb prot5448.

55. Ondov BD, Bergman NH, Phillippy AM. Interactive metagenomic visualization in a web browser. BMC Bioinformatics. 2011:12:385.

56. Schubert M, Lindgreen S, Orlando L. AdapterRemoval v2: rapid adapter trimming, identification, and read merging. BMC Res Notes. 2016;9:88.

57. Schubert M, Ginolhac A, Lindgreen S, Thompson JF, Al-Rasheid KA,

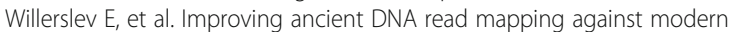
reference genomes. BMC Genomics. 2012;13:178.

58. Li H, Handsaker B, Wysoker A, Fennell T, Ruan J, Homer N, et al. The sequence alignment/map format and SAMtools. Bioinformatics. 2009;25(16): 2078-9.

59. Okonechnikov K, Conesa A, Garcia-Alcalde F. Qualimap 2: advanced multisample quality control for high-throughput sequencing data. Bioinformatics. 2016;32(2):292-4.

60. Schmieder R, Edwards R. Quality control and preprocessing of metagenomic datasets. Bioinformatics. 2011;27(6):863-4.

61. Altschul SF, Gish W, Miller W, Myers EW, Lipman DJ. Basic local alignment search tool. J Mol Biol. 1990;215(3):403-10

\section{Publisher's Note}

Springer Nature remains neutral with regard to jurisdictional claims in published maps and institutional affiliations.

Ready to submit your research? Choose BMC and benefit from

- fast, convenient online submission

- thorough peer review by experienced researchers in your field

- rapid publication on acceptance

- support for research data, including large and complex data types

- gold Open Access which fosters wider collaboration and increased citations

- maximum visibility for your research: over $100 \mathrm{M}$ website views per year

At BMC, research is always in progress.

Learn more biomedcentral.com/submissions 